\title{
Universality of the island density exponent in growth models of monomer and dimer chains in two dimensions
}

\author{
K. Mazzitello, ${ }^{1}$ J. L. Iguain, ${ }^{1}$ C. M. Aldao, ${ }^{2}$ and H. O. Mártin ${ }^{1}$ \\ ${ }^{1}$ Physics Department, School of Exact and Natural Sciences, Universidad Nacional de Mar del Plata, Deán Funes 3350, \\ 7600 Mar del Plata, Argentina \\ ${ }^{2}$ Institute of Materials Science and Technology (INTEMA), Universidad Nacional de Mar del Plata-CONICET, \\ Juan B. Justo 4302, 7600 Mar del Plata, Argentina
}

(Received 15 September 1999)

\begin{abstract}
Using Monte Carlo simulations, the scaling of the island density exponent for the aggregation of particles as chains of monomers and dimers is examined. We found that particles can perform a one- or two-dimensional diffusion depending on the details of the models and on the values of the coverage and diffusion anisotropy. Hence a nonuniversal behavior for the island density exponent $\chi$ can be obtained. Eventually, in the asymptotic regime, the one-dimensional exponent $(\chi=1 / 4)$ is always recovered because the formed one-dimensional islands (chains) play the role of obstacles to the diffusing particles which are thus forced to perform a one-dimensional diffusion.
\end{abstract}

PACS number(s): 61.43.Bn, 68.35.Fx, 05.40.-a

\section{INTRODUCTION}

Several models of deposition, diffusion, and irreversible aggregation of particles forming immobile islands on twodimensional square lattices have been recently studied [1-9]. The growth of islands is governed by the ratio $R$ between the average number of jumps per unit of time for an isolated particle and the average number of deposited particles per site and unit of time. When $R$ increases, each adatom performs a greater number of hops on average between the incoming of particles. Then adatoms have a greater probability of reaching already existing islands than forming new ones. More specifically, at a fixed coverage $\theta$, it has been shown that the average number of island per lattice site, $N$, behaves as $N \sim R^{-\chi}$ for large enough $R$. The so-called "island density exponent" $\chi$ is expected to be $\chi=1 / 4$ for deposited particles performing a one-dimensional diffusion (which is equivalent to working with a one-dimensional substrate) and $\chi=1 / 3$ for two-dimensional diffusion.

An interesting question arises when the diffusion is anisotropic. Let $A$ be the anisotropic parameter defined as $A$ $=P_{y} / P_{x}$, where $P_{y}$ and $P_{x}$ are the probabilities of jumping per time unit of an isolated particle between two firstneighbor sites of a square lattice in the $y$ and $x$ directions, respectively. Monte Carlo results obtained for point, compact, and approximately square islands strongly suggest that $\chi=1 / 3$ for $1 \leqslant A<\infty[5,9]$. Then, for finite values of $A$, the exponent $\chi$ seems to be universal (i.e., $A$ independent) and would correspond to the case of two-dimensional diffusion. For the case of point islands, only for infinite anisotropic diffusion, $A=\infty$, is the one-dimensional result $\chi=1 / 4$ recovered.

Very recently, two similar growth models of onedimensional islands in a square lattice were studied $[10,11]$. In one of them, islands form as chains of monomers and in the other as chains of dimers. In both models particles aggregate at the ends of the chains and the probability of sticking is zero at the lateral sides. Let us assume that the growth direction in both models is the $x$ direction. Lateral sides, then, play the role of obstacles for the diffusion of particles in the $y$ direction. This blocking, caused by the formed islands, affects subsequent particle aggregation and its effects are stronger for $A \gg 1$ (i.e., $P_{y} \gg P_{x}$ ) than for isotropic diffusion. Blocking also appears to be crucial for monomer islands causing $\chi$ to smoothly change from $1 / 3$ to $1 / 4$ as $A$ increases from $A=1$ to the limit $A \rightarrow \infty$ (provided that $A$ is finite, $A<\infty)$. Particles perform an "effective" onedimensional diffusion in the $x$ direction (i.e., in the direction perpendicular to the fast diffusion direction). This nonuniversal behavior contrasts with the Monte Carlo results obtained for a dimer-chain aggregation model [10]. In this case, we found that $\chi=1 / 3$ for $1 \leqslant A<\infty$.

The results of Refs. [10] and [11] were both obtained within the same range of $R\left(10^{7}<R<10^{12}\right)$. A natural question arises from these results: what is responsible for obtaining very different behaviors using two models that are so similar. The first goal of the present work is to answer this question.

The second question we address is related to universality. Both models behave in different ways within a large region of $R$, but in the limit $R \rightarrow \infty$ one expects a unique $\chi$ for both models, recovering a universal behavior. We found that this exponent is $\chi=1 / 4$, which corresponds to the onedimensional case. This outcome is valid in the whole range of the anisotropic parameter $A$. As it will be described in Sec. III, $\chi$ adopts the value $1 / 4$ for any $A(1 \leqslant A<\infty)$. Since the average length of the islands increases as $R$ increases, particles have multiple collisions with the lateral sides of the islands already formed, independently of the value adopted for $A$. Hence the arriving particles perform an effective onedimensional diffusion before attaching to islands ends. We will show that these arguments are independent of the details of the models as long as we deal with one-dimensional islands.

\section{MODELS}

As commented on above, we studied two growth models of one-dimensional islands. In model I these islands are com- 
posed of monomers and in model II of dimers. In both models the substrate is represented by a square lattice of $L_{x}$ $\times L_{y}$ sites where the distance between nearest neighbor (NN) sites is $a$. Periodic boundary conditions were adopted in order to avoid edge effects. Once deposited, particles describe random walks and form islands. Diffusion can be anisotropic; i.e., particles can have different probabilities of jumping to a NN site in the $x$ than in the $y$ direction. We will assume that islands can neither break nor diffuse.

In both models the following processes take place: (a) deposition, (b) diffusion, (c) nucleation, and (d) aggregation. The rules for model I are as follows.

(a) Deposition: each empty site of the lattice is occupied by a new particle with probability $\varepsilon$ per unit of time $t$.

(b) Diffusion: an isolated particle (i.e., a particle not bounded to an island) attempts to jump to any of its NN sites in the $x$ direction with probability $P_{x}$ and to any of its NN sites in the $y$ direction with probability $P_{y}$, per unit of time $t$. If the particle attempts to jump to an occupied site, the jump is not performed and the particle remains at its original site.

(c) Nucleation: if, as a consequence of diffusion or deposition, a particle arrives at a NN site in the $x$ direction of a second isolated particle, these two particles nucleate, forming a new island composed of two particles.

(d) Aggregation: if, as a consequence of diffusion or deposition, a particle arrives at a NN site in the $x$ direction of an island, this particle sticks to this island, increasing the number of particles in the island by 1 .

The rules of deposition and diffusion for model II are the same as for model I. The rest of the rules for model II are as follows.

$\left(c^{\prime}\right)$ Nucleation: if, as a consequence of diffusion or deposition, a particle arrives at a NN site in the $y$ direction of a second isolated particle, these two particles nucleate, forming a dimer. This dimer plays the role of a seed for the dimer chain.

$\left(d^{\prime}\right)$ Aggregation: Fig. 1 shows growth sites for different structures. If a diffusing or deposited particle arrives at a growth site, it sticks, increasing the number of particles of this island by 1 .

In short, the only difference between the two models is the manner in which particles stick. In model I, particles located at nearest neighbors in the $x$ direction become bounded and form islands. In model II, particles must form dimers first. This occurs when two isolated particles meet as nearest neighbors in the $y$ direction. Then dimers can grow by incorporating particles and forming new dimers in the $x$ direction.

\section{RESULTS AND DISCUSSION}

As commented on in Sec. I, the Monte Carlo results obtained from models I and II are quite different (Refs. [10] and [11]). A nonuniversal behavior of the exponent $\chi$ as a function of the anisotropic parameter $A$ for model I and a universal behavior independent of the value of $A$ (for $1 \leqslant A$ $<\infty$ ) for model II were found. Moreover, the results of models I and II were both obtained approximately in the same region of values of $R$. Coverages used were $\theta=0.05$ for model I and $\theta=0.10$ for model II. These values of $\theta$ are

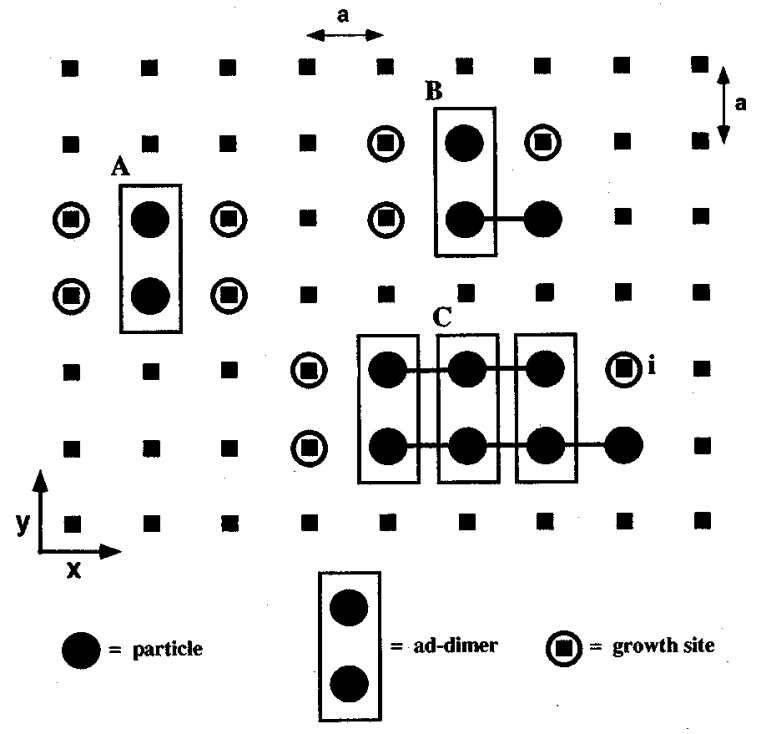

FIG. 1. The substrate (solid squares) and the growth of dimer chains. Two particles that become nearest neighbors in the $y$ direction nucleate, forming a dimer as shown in structure $A$. This dimer plays the role of a seed for a dimer chain. Next, particles can aggregate in growth sites as seen in structure $B$ to finally form a chainlike structure $C$.

comparable because in model II the islands are composed of dimers and then it is needed to double the number of particles to obtain the same number of islands of similar lengths. Indeed, in our Monte Carlo simulations approximately the same number of islands with the same average length of islands were obtained.

We will now attempt to explain the reason for the discrepancy found between models I and II. Let us start by defining a growth site for an island as a site where a monomer can attach to this island. These growth sites are located at the ends of islands for both models. Figure 1 shows growth sites (open circles), particles (solid circles), and dimers in model II. Assuming $A \gg 1$ (i.e., $P_{x} \ll P_{y}$ ), it is very difficult for a monomer, diffusing from the bottom, to reach the growth site $i$ and stick to island $C$. The site $i$ plays the role of a hidden growth site for monomers arriving from the bottom. Then a monomer can pass through the end of an island without attaching to it.

The situation described above never occurs in the case of islands composed of monomers (model I). When a monomer reaches the end of an island, it always sticks to this end. A monomer between two long islands, especially if $A \gg 1$, collides many times before reaching an island end (i.e., it performs an effective one-dimensional diffusion in the $x$ direction). Eventually, when the monomer passes close to an island end, it always sticks to this island in model I. Conversely, for model II, depending on the island ending, the monomer can escape from the region limited by the two parallel island and perform a two-dimensional diffusion.

In order to simulate the case of hidden growth sites which appear in model II, we modified model I by adding a new rule: a monomer arriving at a growth site sticks to the island with probability $p$. If the monomer does not stick, the monomer will continue diffusing.

Figure 2 shows $N$ versus $R$ in $\log$-log scales for the modi- 


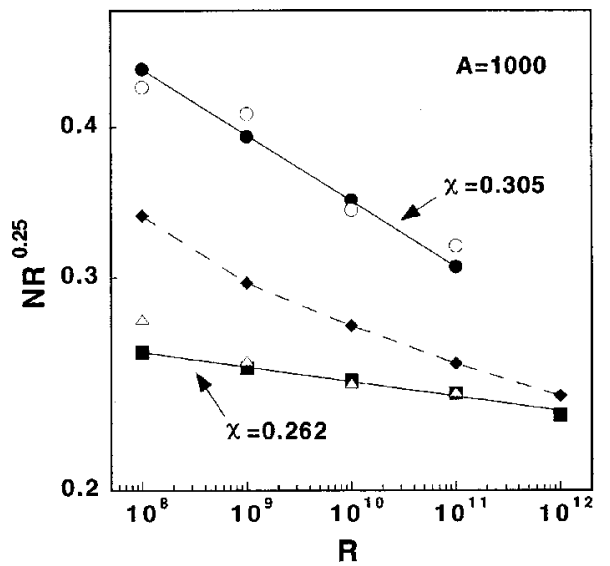

FIG. 2. The island density for model I (monomer chains) with different sticking probability $p$ and for model II (dimer chains) as a function of $R$ : Solid squares correspond to model I with $p=1$, open triangles to $p=1 / 10$, solid diamonds to $p=1 / 50$, and solid circles to $p=1 / 200$. Open circles correspond to model II. Coverages are $\theta$ $=5 \%$ for model I and $\theta=10 \%$ for model II.

fied model I with $A=10^{3}$ and for various values of the sticking probability $p$. Let us compare first the case of $p=1 / 200$ with, the case of $p=1$. As is shown, $\chi$ for $p=1 / 200$ is greater than for $p=1$ in the examined range of $R$. If this behavior holds for larger values of $R$ than those shown, the curves must cross. However, this is not expected to happen because the density of islands for $p<1$ must be always greater or equal than for the case corresponding to $p=1$ regardless of $R$. In general, the possible effects of $p$ being different from 1 in the island formation must decrease as particle diffusion becomes higher, i.e., as $R$ increases. Then we conclude that these curves must eventually match, and then they will present the same exponent $\chi$ as $R \rightarrow \infty$. It will be seen below that, for large enough values of $R, \chi \rightarrow 1 / 4$ for model I regardless of $p$. This tendency is apparent for values of $p$ greater than $1 / 200$, for example, for $p=1 / 50$, as also shown in Fig. 2. For $p=1 / 10$ the matching of curves already appears in the region of $R$ shown in this figure.

The matching curves in Fig. 2 correspond to similar structures because $N$ and the separation between islands in the $y$ direction (equal to $1 / \theta$ ) are the same. For $p<1$, the diffusing particles do not stick to the first end of an island that they find. Thus the average number of steps performed by an isolated particle with $p<1$ is greater than for the case of $p$ $=1$. For small values of $p$, then, it is more likely for an isolated particle to find another diffusing isolated particle and create a new island. Hence the number of islands (and the density) for $p<1$ must be greater or equal than for the case corresponding to $p=1$. The results of model II with $A$ $=10^{3}$ are also shown in Fig. 2. These results are very similar to those obtained for the modified model I with $p=1 / 200$, giving support to the above-mentioned argument used to explain the discrepancy between models I and II.

We will focus now on the universality of the island density exponent $\chi$ for model I. In this model islands are linear chains with a width of only one particle. Since the islands are randomly distributed, the average distance between two consecutive islands in the $y$ direction is $1 / \theta$. This distance remains fixed in the limit $R \rightarrow \infty$, as long as the coverage is kept constant. Since for large values of $R$ the number of

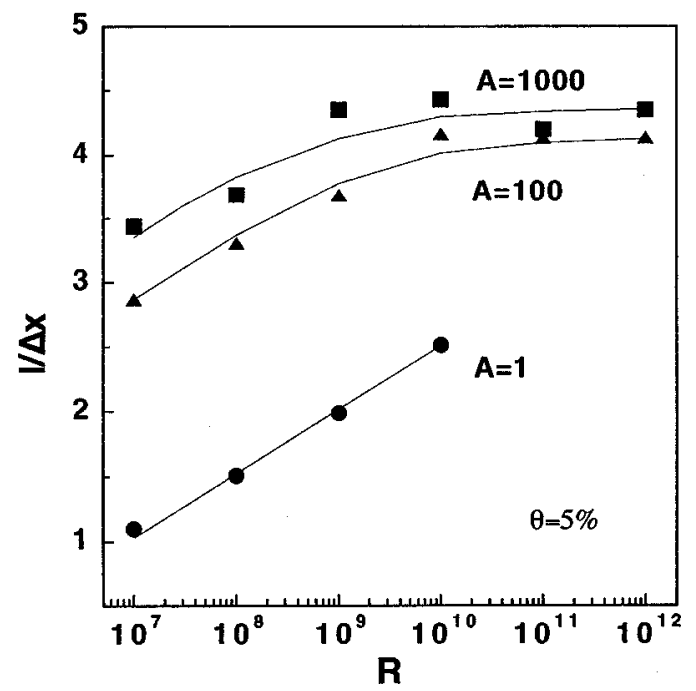

FIG. 3. The ratio between the average length of islands $l$ and the square root of the mean-square displacement in the $x$ direction, $\Delta x$, as a function of $R$ for different values of the anisotropic parameter $A$. Note that $\Delta x$ is always smaller than $l$ and $\Delta x / l$ decreases with $R$ and $A$, indicating a tendency towards a one-dimensional diffusion. (Lines are a guide to the eye.)

monomers is much smaller than the number of islands, we can write $\theta \cong N l$, where $l$ is the average length of islands. As a consequence $l \sim R^{\chi}$. As monomers do not stick to the lateral side of islands, their diffusion is limited to rectangles of length in the order of $l$. When $R$ increases, $l$ also increases and the diffusion of monomers becomes more and more one dimensional. Unless the square root of the mean-square displacement in the $x$ direction $(\Delta x)$ were much greater than $l(\Delta x \gg l)$, one should expect that monomers perform an effective one-dimensional diffusion in the limit $R \rightarrow \infty$. The mean-square displacement $\Delta x^{2}$ can be computed from the mean value of steps $\bar{n}$ performed by a monomer until its attachment to another monomer or existing chain (i.e., the end of the diffusion due to nucleation or aggregation processes) as follows:

$$
\Delta x(\bar{n})^{2}=\bar{n} \frac{P_{x}}{P_{x}+P_{y}} .
$$

This equation holds because monomers move freely in the $x$ direction before sticking to another particle or an island. In Fig. $3 l / \Delta x$ is plotted as a function of $R$ for $A=1,100$, and 1000 and $\theta=0.05$, where $l$ and $\bar{n}$ were obtained from Monte Carlo simulations and $\Delta x$ using Eq. (1). Note that in all cases $l>\Delta x$. This result strongly indicates that $\chi$ will correspond to a one-dimensional diffusion when $R \rightarrow \infty$.

More information about the way in which monomers diffuse is revealed by computing the square root of the meansquare displacement in the $y$ direction $(\Delta y)$ as a function of jumping step $n$ for an isolated random walker (a tracer particle). The particle starts its walk in a randomly chosen empty site of the substrate. This substrate was previously obtained by applying the rules of model I up to the point where the final coverage $\theta$ is reached. This random walker does not stick at the end of islands, and then it always diffuses. For this tracer particle we also compute the number of 


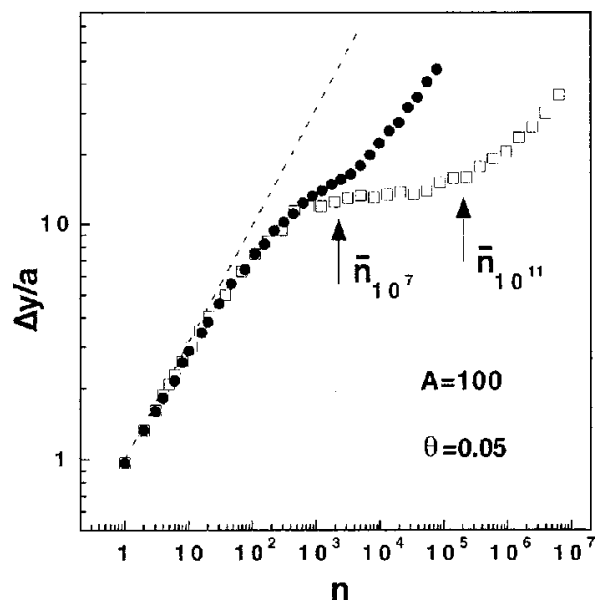

FIG. 4. The square root of the mean-square displacement in the $y$ direction $(\Delta y / a)$ as a function of jumping step $n$ for an isolated random walker, i.e., a tracer particle that does not stick at the end of islands. The dashed straight line corresponds to a random walk on an empty substrate. As diffusion progresses, the tracer particle eventually interacts with islands that limit the displacement in the $y$ direction. Note that, around $\bar{n}$, the random walker remains trapped between islands and then the diffusion becomes one dimensional in the $x$ direction. Open squares correspond to $R=10^{11}$ and solid circles to $R=10^{7}$.

distinct visited sites, $S(n)$, as a function of $n$. Figure 4 shows $\log _{10}(\Delta y / a)$ versus $\log _{10}(n)$ for $A=100, \theta=0.05$, and $R$ $=10^{7}$ and $10^{11}$. The dashed straight line corresponds to a random walk on an empty substrate (a square lattice with no islands). At short times, the random walk behaves as in an empty substrate. As diffusion progresses, the walker eventually finds the lateral side of an island that plays the role of an obstacle delaying the displacement in the $y$ direction. At long times the random walker recovers the behavior $\Delta y \propto t^{1 / 2}$. It is clearly seen that $\Delta y$ is modified by the presence of obstacles and that the effect increases with $R$. This means that the diffusion becomes more one dimensional as $R$ increases. Note that $\bar{n}$ is much smaller than the number of steps needed by a random walk to reach the long-time regime $(\Delta y$ $\left.\propto t^{1 / 2}\right)$. Moreover, $\Delta y(\bar{n}) \sim 1 / \theta$, where $1 / \theta$ is the mean separation between islands. This result clearly shows that, around $\bar{n}$, the random walker remains trapped between islands performing an effective one-dimensional diffusion in the $x$ direction.

The number of different sites visited by the tracer, $S(n)$, is a function of $n$. Specifically, the exponent $\alpha$ is defined through the relation

$$
S(n) \sim \bar{n}^{\alpha} .
$$

In our case, we are interested in $\alpha$ as the local slope of $\log _{10}(S)$ as a function of $\log _{10}(n)$ around the point $\bar{n}$. The exponent $\alpha$ gives us information regarding the kind of diffusion that the random walk performs near $\bar{n}$. If $\alpha=1$, we have a two-dimensional diffusion and expect a two-dimensional island density exponent $\chi=1 / 3$. On the other hand, if $\alpha$ $=1 / 2$, we expect $\chi=1 / 4$, corresponding to one-dimensional diffusion. The values of the exponent $\alpha$ as a function of $R$ for

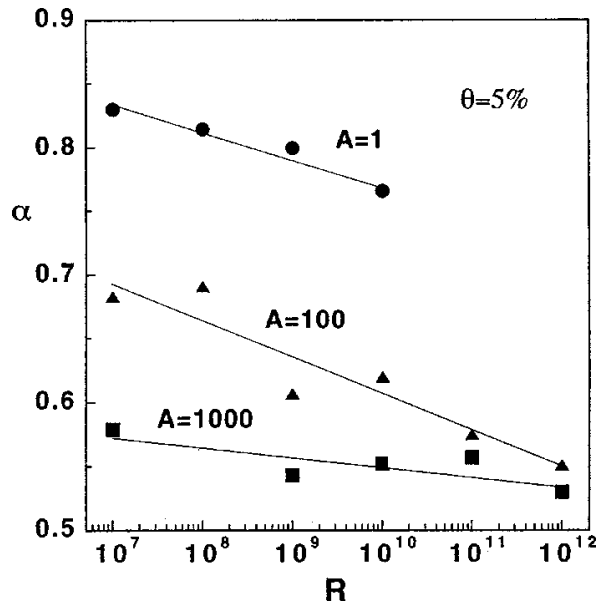

FIG. 5. Values of exponent $\alpha$ as a function of $R$ and $A$. The decrease of $\alpha$ with $R$ and $A$ indicates that the diffusion becomes more one dimensional.

$A=1,100$, and 1000 are presented in Fig. 5, showing that $\alpha$ clearly decreases with $R$, indicating that the diffusion becomes more one dimensional.

All the results previously shown correspond to $\theta=0.05$. If $\theta$ changes, the average vertical distance between islands changes, because this distance is $1 / \theta$. For a fixed value of $R$, the average length of islands $l$ also changes. It is expected that $l$ increases with $\theta$, and this indeed occurs. Then, for a larger value of $\theta$, the diffusion of monomers in the $y$ direction becomes more limited because the islands are closer to each other and also they are longer. For these reasons we expect that the exponent $\chi$ will approach $1 / 4$ when $\theta$ increases. In Fig. 6 we present $\log _{10}\left(N R^{1 / 4}\right)$ versus $\log _{10}(R)$ for $A=100$ and $\theta=0.02,0.05$, and 0.10 . It can be observed that $\chi$ decreases with $\theta$, and the results suggest a crossover to $\chi$ $=1 / 4$ for larger values of $R$ than those shown in the figure. Also, from these results we conclude that for non-extremelylarge values of $R$, one can find an effective exponent which depends both, on the anisotropic diffusion parameter $A$ and on the coverage $\theta$.

In summary, the results shown in Figs. 3-6 strongly suggest that for model I, $\chi \rightarrow 1 / 4$ when $R \rightarrow \infty$, for $1 \leqslant A<\infty$.

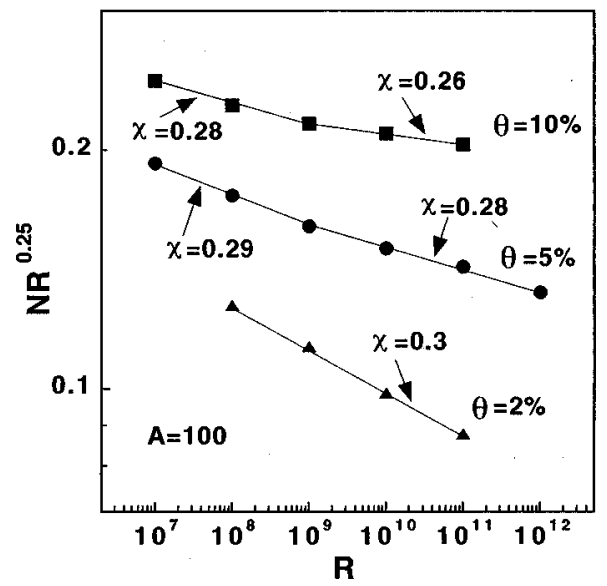

FIG. 6. Island density exponent $\chi$ as a function of $R$ for different coverages. Note that the higher the coverage, the more one dimensional the diffusion is. As coverage increases, the blocking action of the formed islands becomes stronger, confining particles to move only in the $x$ direction. 
Figure 2 shows that the results of $\chi \cong 1 / 3$ obtained for model II can be explained with the modified model I. As mentioned above, the curves of $\log _{10}(N)$ against $\log _{10}(R)$ must match for large values of $R$. Then we conclude that for the modified model I and, in consequence also for model II, it is expected that $\chi \rightarrow 1 / 4$ as $R \rightarrow \infty$, for $1 \leqslant A<\infty$. Then we conclude that universality is recovered for the three models in the asymptotic regime of $R$.

\section{CONCLUSIONS}

We have presented Monte Carlo results for the submonolayer epitaxial growth corresponding to two models in which islands grow as linear chains of monomers and dimers. We focused on the dependence of the island density on the diffusion to the deposition rate $R$ and the diffusion anisotropy $A$, with special interest in the island density exponent $\chi$ corresponding to the asymptotic regime $R \rightarrow \infty$.

In short, in the asymptotic regime, we conclude that the one-dimensional exponent $\chi=1 / 4$ is obtained because we are dealing with one-dimensional islands (chains) that play the role of obstacles to the diffusing particles, which are forced to perform a one-dimensional diffusion. For values of $R$ not large enough, these obstacles cannot determine the effective one-dimensional character of diffusion, and then particles can perform a one- or two-dimensional diffusion depending on the details of the models and on the values of the coverage $\theta$ and diffusion anisotropy $A$. Thus a nonuniversal behavior of the exponent $\chi$ can be found.

\section{ACKNOWLEDGMENTS}

This work was partially supported by the National Council for Scientific and Technical Research (CONICET) of Argentina. K.M. acknowledges financial support of FOMEC (Argentina). One of us (H.O.M.) would like to acknowledge the kind hospitality of the Abdus Salam International Center for Theoretical Physics (Trieste, Italy) where this work was partially written.
[1] A. D. King and D. P. Woodruff, Growth and Properties of Ultrathin Epitaxial Layers (Elsevier, Amsterdam, 1997).

[2] A.-L. Barabási and H. E. Stanley, Fractals Concepts in Surface Growth (Cambridge University Press, Cambridge, England, 1995).

[3] A. Pimpinelli and J. Villain, Physics of Crystal Growth (Cambridge University Press, Cambridge, England, 1998).

[4] P. Jensen, A.-L. Barabási, H. Larralde, S. Havlin, and H. E. Stanley, Phys. Rev. B 50, 15316 (1994).

[5] M. C. Bartlet and J. W. Evans, Phys. Rev. B 46, 12675 (1992).
[6] J. G. Amar, F. Family, and Pui-Man Lam, Phys. Rev. B 50, 8781 (1994).

[7] F. Family and J. G. Amar, Mater. Sci. Eng., B 30, 149 (1995).

[8] J. G. Amar and F. Family, Thin Solid Films 272, 208 (1996).

[9] T. R. Linderoth, J. J. Mortensen, K. W. Jacobsen, E. Lægsgaard, I. Stensgaard, and F. Besenbacher, Phys. Rev. Lett. 77, 87 (1996)

[10] J. L. Iguain, H. O. Mártin, and C. M. Aldao, Phys. Rev. B 59, 4596 (1999).

[11] K. I. Mazzitello, J. L. Iguain, and H. O. Mártin, J. Phys. A 32, 4389 (1999). 